\title{
Intrinsic Coupling between Gamma Oscillations, Neuronal Discharges, and Slow Cortical Oscillations during Human Slow-Wave Sleep
}

\author{
Sarang S. Dalal, ${ }^{1,2}$ Carlos M. Hamamé, ${ }^{1,2}$ Jean-Baptiste Eichenlaub, ${ }^{1,2,3}$ and Karim Jerbi ${ }^{1,2}$ \\ ${ }^{1}$ INSERM U821, Dynamique Cérébrale et Cognition, 69675 Lyon, France, ${ }^{2}$ Université Claude Bernard, Lyon I, 69622 Villeurbanne, France, and ${ }^{3}$ École \\ Doctorale Neurosciences et Cognition, Université Claude Bernard, Lyon I, 69622 Villeurbanne, France \\ Review of Le Van Quyen et al.
}

In the first electrocorticograms, Richard Caton (1877) connected a mirror galvanometer to exposed rabbit brains and observed slow sleep-related fluctuations. Several decades later, Loomis et al. (1937) applied the then-fledgling technique of scalp EEG to classify sleep stages in humans, observing dominant low-frequency oscillations during what is now called slow-wave sleep (SWS).

More than a century after Caton's (1877) initial findings, the mechanism and significance of slow oscillations in the brain remain important subjects of research. A growing body of literature associates slower oscillations in general (below $\sim 10 \mathrm{~Hz}$ ) to neuronal spiking activity. Slow waves during deep sleep, with frequencies $<1 \mathrm{~Hz}$, are now known to reflect alternations between depolarizing UP states that result in widespread unit firing, and hyperpolarizing DOWN states that result in relative neuronal silence (Steriade et al., 1993).

Faster rhythmic activity of $\sim 30-200$ $\mathrm{Hz}$, often referred to as the gamma band,

Received Aug. 16, 2010; revised Sept. 7, 2010; accepted Sept. 9, 2010.

This work was supported by a Marie Curie Fellowship to S.S.D. from the European Commission, a grant to C.M.H. from the Fondation Fyssen, and a grant to K.J. from the Fondation pour la Recherche Médicale. We thank Dr. Jean-Philippe Lachaux and Dr. Perrine Ruby for helpful discussions.

Correspondence should be addressed to Dr. Sarang $S$. Dalal, INSERM U821, CH Le Vinatier, 95 Boulevard Pinel, Bât 452, 69675 Lyon, France. E-mail: sarang.dala|@inserm.fr.

DOI:10.1523/JNEUROSCI.4275-10.2010

Copyright $\odot 2010$ the authors $\quad 0270-6474 / 10 / 3014285-03 \$ 15.00 / 0$ has been linked to a variety of active cognitive processes, including attention, sensory processing, perceptual binding, and even conscious experience (Jensen et al., 2007). Furthermore, gamma-band activity appears to be the most reliable electrophysiological correlate of the fMRI blood oxygenation level-dependent response (Lachaux et al., 2007), and its coupling to both slower oscillations (Canolty et al., 2006) and unit activity (Whittingstall and Logothetis, 2009) are highly active areas of research.

Le Van Quyen et al. (2010) extend this line of research by examining the intensity and timing of gamma events with respect to sleep stages and slow oscillations in humans. Scalp EEG was simultaneously acquired with local field potentials (LFPs) and multiunit activity (MUA) recordings via macroelectrodes and microelectrodes implanted in epileptic patients. Gamma oscillations $(40-120 \mathrm{~Hz})$ appeared across all sleep stages in the human cortex, yet, surprisingly, they occurred far more frequently during SWS than during quiet wakefulness or rapid eye movement (REM) sleep [Le Van Quyen et al. (2010), their Fig. 2A]. Parahippocampal gyrus appeared to be the origin for many of these events, although they were observed to a lesser extent across all other implanted areas, including cingulate, occipital, orbitofrontal, and superior temporal cortices, as well as supplementary motor area [Le Van
Quyen et al. (2010), their Fig. 3]. Additionally, hippocampal ripples $(130-250 \mathrm{~Hz})$ moderately increased the likelihood of gamma event detection in parahippocampal gyrus within the subsequent $100 \mathrm{~ms}$ [Le Van Quyen et al. (2010), their Fig. 5], contributing evidence that SWS may facilitate memory consolidation from hippocampus to neocortex.

Another principal finding by Le Van Quyen et al. (2010) was that LFP gamma events and multiunit discharges during deep sleep correlated specifically with the positive peaks of slow oscillations recorded on the scalp [Le Van Quyen et al. (2010), their Figs. 1, 2E]. In turn, multiunit discharges were highly concentrated with the troughs of LFP gamma oscillations [Le Van Quyen et al. (2010), their Fig. 7]. These multiscale coupling relationships were also observed for K-complexes [Le Van Quyen et al. (2010), their supplemental Fig. 1, available at www.jneurosci.org as supplemental material], which resemble single cycles of slow waves and occur primarily during sleep stage 2. Therefore, the UP state of sleep slow waves coupled to MUA bursts as well as to high-frequency oscillations, alternating with DOWN states corresponding to global reductions in activity. These important findings provide strong evidence in humans supporting the UP/DOWN state hypothesis of Steriade et al. (1993).

Csercsa et al. (2010) recently described another microelectrode study in sleeping 
epileptic patients showing similar multiscale coupling on the cortical surface of frontal, temporal, and parietal structures during SWS. Furthermore, these effects localized to cortical layers I-III, suggesting generation by the apical dendrites of pyramidal cells. Using similar methods, the same laboratory previously examined surfacenegative K-complexes, which strongly correlated with DOWN states also involving layers I-III (Cash et al., 2009). Their conclusion that the K-complex therefore exclusively reflects isolated DOWN states is seemingly contradicted by Le Van Quyen et al. (2010). However, on further examination of their results (Cash et al., 2009, their Fig. 3), many locations near the surface of frontal cortex did indeed exhibit increases in MUA and gamma oscillations. Bearing in mind that Le Van Quyen et al. (2010) exclusively recorded at depth, with the most robust effects observed in parahippocampal gyrus, these studies may in fact provide converging evidence that the $\mathrm{K}$-complexes of sleep stage 2 might be isolated single cycles of slow waves, resulting in widespread transient DOWN states, followed by UP states in more specific structures.

An intriguing mechanism for these coupling relationships is suggested by another recent article. Fröhlich and McCormick (2010) developed an innovative "fieldclamp" technique to mimic endogenous electric fields such as slow waves; with it, their results suggest that endogenous fields are sufficiently strong to not only directly induce alternating UP/DOWN states, but also to reinforce and spread existing slow rhythms.

Indeed, transcranial magnetic stimulation (TMS) pulses induce prominent slow oscillations when applied during sleep stage 2 and SWS, but not during sleep stage 1 or wakefulness (Massimini et al., 2007). These induced oscillations behave as traveling waves arising from the TMS focus, similar to naturally occurring slow waves. This implies that cortex may be in a peculiarly excitable state during the deeper stages of non-REM sleep and supports the plausibility of focal generators that initiate the waves, which then propagate across the entire cerebral cortex primarily through local connections or endogenous field effects rather than longrange connections. These could manifest as isolated $\mathrm{K}$-complexes as the cortex transitions to a more susceptible state during sleep stage 2, evolving into selfsustaining slow waves during SWS as the cortex reaches maximum excitability.

One potential function of SWS and gamma/ripple oscillations is to facilitate declarative memory consolidation involving the hippocampus (Wierzynski et al., 2009), though the precise mechanism remains unclear. Le Van Quyen et al. (2010) suggest that their results support the hypothesis that hippocampus ripples entrain neocortical gamma oscillations during SWS and thereby drive memory consolidation, in agreement with Wierzynski et al. (2009), who found prefrontal cortex unit firing $100 \mathrm{~ms}$ after hippocampal ripples in the rat during SWS. In a human macroelectrode sleep study, Axmacher et al. (2008) found that rhinal cortex gamma events were indeed associated with memory consolidation; however, they followed hippocampal ripples by only $1 \mathrm{~ms}$ on average, suggesting information flow originating from a common third structure. Finally, several studies in rats have shown that hippocampus ripples follow entorhinal UP states by 50-200 ms (e.g., Hahn et al., 2006), suggesting that it is instead neocortex that drives hippocampus during SWS. Perhaps more sophisticated measures of directionality such as Granger causality may clarify these inconsistencies.

The findings of Le Van Quyen et al. (2010) add to the controversy regarding the nature of both gamma oscillations themselves and their relationship to sleep stages. Another recent study with macroelectrodes implanted in epilepsy patients showed a similar relation between sleep stage and the prevalence of $>80 \mathrm{~Hz}$ ripple events (Bagshaw et al., 2009), whereas several previous studies show significantly decreased gamma power during SWS relative to wakefulness (Gross and Gotman, 1999; Cantero et al., 2004; Axmacher et al., 2008).

Methodological differences of Le Van Quyen et al. (2010) may explain some of these discrepancies. One key difference was the use of microelectrodes, rarely implanted in human patients, which sample smaller volumes than implanted macroelectrodes. This suggests that larger neuronal ensembles may synchronize in the gamma band during wakefulness, but smaller populations are involved during SWS. Additionally, rather than calculating average gamma power over long intervals, the analyses calculated the rate of individual gamma events and examined the coupling between gamma power and the phase of slow waves. Gamma events associated with cognition and perception during wakefulness might have also been underestimated due to the selection criteria of $>100 \mathrm{~ms}$ duration as well as visual identification of oscillations on raw elec- trode traces; these types of responses are often relatively weak and brief, requiring repeated trials and statistical analyses for reliable detection, and so may not necessarily resemble obvious oscillations in continuous recordings. Finally, several sleep studies, including the one by Le Van Quyen et al. (2010), define "wakefulness" as the period immediately before entering sleep stage 1 in a dark room with no external stimulation, and therefore findings may not directly apply to fully alert wakefulness.

It would be particularly interesting to clarify the role of gamma oscillations with respect to the two subdivisions of REM sleep. Phasic REM sleep is characterized by the eye movements themselves, but comprises only 5-30\% of total REM sleep (Hansotia et al., 1990), with tonic REM sleep and its associated generalized atonia accounting for the remainder. Human intracranial EEG has shown that cortical gamma power is in fact enhanced during phasic but not tonic REM sleep (Gross and Gotman, 1999). Likewise, rat hippocampus exhibits increased gamma synchrony during phasic REM sleep, while gamma power and unit firing rates decrease during tonic REM sleep (Montgomery et al., 2008). Therefore, significant gamma power may be present only during relatively short phasic periods, explaining the relatively low gamma-REM association found by Le Van Quyen et al. (2010).

The results by Le Van Quyen et al. (2010) are nevertheless compelling in the context of the proposed role of gamma oscillations during wakefulness, reinforcing the concept of sleep as a paradoxically active brain state in which gamma oscillations may participate in long-hypothesized sleep functions such as memory consolidation and learning. The reported predominance of spontaneous gamma events during the deeper stages of sleep does not necessarily preclude a significant role for gamma-band activity in conscious processing. Indeed, task-related increases of gamma activity have been associated with attention, memory, and perceptual awareness (Jensen et al., 2007; Wyart and TallonBaudry, 2009). More generally, elucidating the role of widespread spontaneous gamma bursts during SWS might benefit from recent evidence for transient attention-related suppressions of broadband gamma activity in the default-mode network during wakefulness (e.g., Jerbi et al., 2010). Incidentally, default mode network structures are thought to play a key role in the generation and propagation of sleep slow waves (Dang-Vu et al., 2008). Therefore, investi- 
gating the relationship between concurrent gamma increases and decreases in large-scale networks and their relationship with slow oscillations and neuronal firing across different levels of vigilance will be critical in future research. Further experiments combining scalp-level EEG, LFP, and unit recordings across multiple sites, as reported here by Le Van Quyen et al. (2010), are poised to play a key role in this endeavor.

\section{References}

Axmacher N, Elger CE, Fell J (2008) Ripples in the medial temporal lobe are relevant for human memory consolidation. Brain 131:1806-1817.

Bagshaw AP, Jacobs J, LeVan P, Dubeau F, Gotman J (2009) Effect of sleep stage on interictal highfrequency oscillations recorded from depth macroelectrodes in patients with focal epilepsy. Epilepsia 50:617-628.

Canolty RT, Edwards E, Dalal SS, Soltani M, Nagarajan SS, Kirsch HE, Berger MS, Barbaro NM, Knight RT (2006) High gamma power is phase-locked to theta oscillations in human neocortex. Science 313:1626-1628.

Cantero JL, Atienza M, Madsen JR, Stickgold R (2004) Gamma EEG dynamics in neocortex and hippocampus during human wakefulness and sleep. Neuroimage 22:1271-1280.

Cash SS, Halgren E, Dehghani N, Rossetti AO, Thesen T, Wang C, Devinsky O, Kuzniecky R, Doyle W, Madsen JR, Bromfield E, Erõss L, Halász P, Karmos G, Csercsa R, Wittner L, Ulbert I (2009) The human K-complex represents an isolated cortical down-state. Science 324:1084-1087.

Caton R (1877) Interim report on the investiga- tion of the electric currents of the brain. BMJ [Suppl 1] :62-65.

Csercsa R, Dombovári B, Fabó D, Wittner L, Erõss L, Entz L, Sólyom A, Rásonyi G, Szucs A, Kelemen A, Jakus R, Juhos V, Grand L, Magony A, Halász P, Freund TF, Maglóczky Z, Cash SS, Papp L, Karmos G, et al. (2010) Laminar analysis of slow wave activity in humans. Brain 133: 2814-2829.

Dang-Vu TT, Schabus M, Desseilles M, Albouy G, Boly M, Darsaud A, Gais S, Rauchs G, Sterpenich V, Vandewalle G, Carrier J, Moonen G, Balteau E, Degueldre C, Luxen A, Phillips C, Maquet P (2008) Spontaneous neural activity during human slow wave sleep. Proc Natl Acad Sci U S A 105:15160-15165.

Fröhlich F, McCormick DA (2010) Endogenous electric fields may guide neocortical network activity. Neuron 67:129-143.

Gross DW, Gotman J (1999) Correlation of high-frequency oscillations with the sleepwake cycle and cognitive activity in humans. Neuroscience 94:1005-1018.

Hahn TT, Sakmann B, Mehta MR (2006) Phaselocking of hippocampal interneurons' membrane potential to neocortical up-down states. Nat Neurosci 9:1359-1361.

Hansotia P, Broste S, So E, Ruggles K, Wall R, Friske M (1990) Eye movement patterns in REM sleep. Electroencephalogr Clin Neurophysiol 76:388-399.

Jensen O, Kaiser J, Lachaux JP (2007) Human gamma-frequency oscillations associated with attention and memory. Trends Neurosci 30, 317-324.

Jerbi K, Vidal JR, Ossandon T, Dalal SS, Jung J, Hoffmann D, Minotti L, Bertrand O, Kahane P, Lachaux JP (2010) Exploring the electrophysiological correlates of the default-mode network with intracerebral EEG. Front Syst Neurosci 4:27.
Lachaux JP, Fonlupt P, Kahane P, Minotti L, Hoffmann D, Bertrand O, Baciu M (2007) Relationship between task-related gamma oscillations and BOLD signal: New insights from combined fMRI and intracranial EEG. Hum Brain Mapp 28:1368-1375.

Le Van Quyen M, Staba R, Bragin A, Dickson C, Valderrama M, Fried I, Engel J (2010) Largescale microelectrode recordings of highfrequency gamma oscillations in human cortex during sleep. J Neurosci 30:7770-7782.

Loomis A, Harvey E, Hobart G (1937) Cerebral states during sleep, as studied by human brain potentials. J Exp Psychol 21:127-144.

Massimini M, Ferrarelli F, Esser SK, Riedner BA, Huber R, Murphy M, Peterson MJ, Tononi G (2007) Triggering sleep slow waves by transcranial magnetic stimulation. Proc Natl Acad Sci U S A 104:8496-8501.

Montgomery SM, Sirota A, Buzsáki G (2008) Theta and gamma coordination of hippocampal networks during waking and rapid eye movement sleep. J Neurosci 28:6731-6741.

Steriade M, Nuñez A, Amzica F (1993) Intracellular analysis of relations between the slow $(<1 \mathrm{~Hz})$ neocortical oscillation and other sleep rhythms of the electroencephalogram. J Neurosci 13:3266-3283.

Whittingstall K, Logothetis NK (2009) Frequencyband coupling in surface EEG reflects spiking activity in monkey visual cortex. Neuron 64: 281-289.

Wierzynski CM, Lubenov EV, Gu M, Siapas AG (2009) State-dependent spike-timing relationships between hippocampal and prefrontal circuits during sleep. Neuron 61:587-596.

Wyart V, Tallon-Baudry C (2009) How ongoing fluctuations in human visual cortex predict perceptual awareness: baseline shift versus decision bias. J Neurosci 29:8715-8725. 